\title{
ON MARTINGALE APPROXIMATION OF ADAPTED PROCESSES
}

\author{
Hervé Queffélec And Dalibor VolnÝ
}

\begin{abstract}
We show that the existence of a martingale approximation of a stationary process depends on the choice of the filtration. There exists a stationary linear process which has a martingale approximation with respect to the natural filtration, but no approximation with respect to a larger filtration with respect to wich it is adapted and regular. There exists a stationary process adapted, regular, and having a martingale approximation with respect to a given filtration but not (regular and having a martingale approximation) with respect to the natural filtration.
\end{abstract}

1. Introduction. Let $(\Omega, \mathcal{A}, \mu)$ be a probability space with a bimeasurable and measure-preserving transformation $T$. For simplicity we shall suppose that $\mu$ is ergodic, i.e. for $A$ measurable, $A=T^{-1} A$ implies that $\mu(A)=0$ or $\mu(A)=1$. For any measurable function $f,\left(f \circ T^{i}\right)$ is a strictly stationary process. Let $\left(\mathcal{F}_{i}\right)$ be an increasing filtration of sub- $\sigma$-algebras of $\mathcal{A}$ such that $\mathcal{F}_{i}=T^{-i} \mathcal{F}_{0}$. By $\mathcal{F}_{-\infty}$ we denote the intersection of all $\mathcal{F}_{i}$ and by $\mathcal{F}_{\infty}$ the $\sigma$-algebra generated by $\mathcal{F}_{i}, i \in \mathbb{Z}$.

We say that for the process $\left(f \circ T^{i}\right)$ there is a martingale approximation w.r.t. $\left(\mathcal{F}_{i}\right)$ if there exists $m \in L^{2}\left(\mathcal{F}_{0}\right) \ominus L^{2}\left(\mathcal{F}_{-1}\right)$ such that $\frac{1}{\sqrt{n}}\left\|S_{n}(f-m)\right\|_{2} \rightarrow 0$ for $n \rightarrow \infty$ where $S_{n}(g)=\sum_{i=0}^{n-1} g \circ T^{i}$.

This condition is equivalent to Gordin's 1969 condition (cf. [V93]). The martingale approximation, since Gordin's 1969 paper [Go], has been a powerful tool in proving central limit theorems for stationary processes. Most results from 70's appear in the monography [Ha-He], for several more recent contributions we can quote e.g. [DeM], [M-Wo] [Wu-Wo] [Pe-U] [Wu07] [Z-Wo] [V93]. The method has been particularly suitable for the study of processes of $X_{k}=g\left(\ldots, e_{-1}, e_{0}\right)$, where $\left(e_{i}\right)$ is an iid sequence; this class includes the (functionals of) stationary linear processes and an interesting study of limit theorems for this class of processes have been done by W. B. Wu in [Wu05].

Here we shall deal with the problem of the dependence of the approximation on the choice of the filtration. The problem was posed by Gordin in his $1968 \mathrm{Ph} . \mathrm{D}$ thesis but seemingly, except [V09], no results have been published. It has only been known as a folklore theorem, that there exist processes which are deterministic, i.e. measurable w.r.t. the natural filtration, but having a martingale approximation w.r.t. another filtration (cf. [V09]). In [V09] a stationary linear process $\left(X_{i}\right)$ with independent innovations $e_{i}$ has been found such that $\left(X_{i}\right)$ is a martingale difference sequence (a sequence of iid, in fact) but does not admit any martingale approximation w.r.t. the filtration $\left(\mathcal{F}_{i}\right)$ given by the process of innovations. The example, however, is not adapted to the filtration $\left(\mathcal{F}_{i}\right)$. Here we shall treat a more difficult situation, when the process is adapted. 
We shall say that the process $\left(f \circ T^{i}\right)$ is regular w.r.t. a filtration $\left(\mathcal{F}_{i}\right)$ if $f$ is $\mathcal{F}_{\infty}$-measurable and $E\left(f \mid \mathcal{F}_{-\infty}\right)=0$. Let us suppose that the process $\left(f \circ T^{i}\right)$ is adapted w.r.t. $\left(\mathcal{F}_{i}\right)$, i.e. $f$ is $\mathcal{F}_{0}$-measurable. Denote by $\mathcal{G}_{j}=\sigma\left\{f \circ T^{i} \mid i \leq j\right\}$ the natural filtration. Recall that by a stationary linear process $\left(X_{k}\right)$ with innovations $e_{i}$ we understand the process of $X_{k}=\sum_{i \in \mathbb{Z}} a_{i} e_{k-i}$ where $\sum_{i \in \mathbb{Z}} a_{i}^{2}<\infty$.

Proposition 1. There exists an adapted stationary linear process $\left(X_{k}\right)$ such that the $X_{k}$ 's are iid and if $\left(\mathcal{F}_{i}\right)$ is the filtration given by the innovations, there is a $c>0$ such that for every $m \in L^{2}\left(\mathcal{F}_{0}\right) \ominus L^{2}\left(\mathcal{F}_{-1}\right)$

$$
\sup _{m \in L^{2}\left(\mathcal{F}_{0}\right) \ominus L^{2}\left(\mathcal{F}_{-1}\right)} \limsup _{n \rightarrow \infty} \frac{1}{\sqrt{n}}\left\|\sum_{i=0}^{n-1}\left(X_{i}-m\right)\right\|_{2}>c .
$$

The proposition shows that $\left(X_{k}\right)$ is also a stationary linear process with another process $\left(g_{i}=X_{i}\right)_{i}$ of independent innovations, adapted to the natural filtration $\left(\mathcal{G}_{i}\right)$, and for $\left(\mathcal{G}_{i}\right)$ we can get much better approximation properties. We can easily change the process $\left(X_{n}\right)$ (e.g. by adding a coboundary) so that it is not an iid sequence (nor a sequence of martingale differences), having no martingale approximation w.r.t. $\left(\mathcal{F}_{i}\right)$ and having a martingale approximation w.r.t. the natural filtration.

One can guess that the natural filtration will bring the best approximation. This, however, is not the case.

Proposition 2. There exists a process $\left(f \circ T^{i}\right)$ and a filtration $\left(\mathcal{F}_{j}\right)$ such that

(i) $\left(f \circ T^{i}\right)$ is not regular w.r.t. the natural filtration $\left(\mathcal{G}_{i}\right)$,

(ii) there is no martingale approximation w.r.t. the natural filtration $\left(\mathcal{G}_{i}\right)$,

(iii) there is a martingale approximation w.r.t. the filtration $\left(\mathcal{F}_{i}\right)$.

Remark 1. The example proving Proposition 2 is such that $g=E\left(f \mid \mathcal{G}_{-\infty}\right)$ is a function different both from $f$ and from 0 , such that $\left(g \circ T^{i}\right)$ is a nontrivial iid sequence.

In general, we can take the natural filtration $\left(\mathcal{G}_{i}^{(1)}\right)_{i}$ of the process $\left(E\left(f \mid \mathcal{F}_{-\infty}\right) \circ\right.$ $\left.T^{i}\right)$, then of the process $\left(E\left(f \mid \mathcal{G}_{-\infty}^{(1)}\right) \circ T^{i}\right), \ldots$ and get an ordered set of $\sigma$-algebras $\mathcal{G}_{-\infty}^{(\alpha)}, \alpha<\alpha_{0}$, where $\alpha_{0}$ is a countable ordinal number, cf. [V85] (in the exemple proving Proposition 2, the process stops already after the second step). For such "cascades" of filtrations we can have martingale approximations and limit theorems similarly as in the case of single filtrations (see [V92]).

Remark 2. There exists a "deterministic process" $\left(f \circ T^{i}\right)$ such that $f \circ T^{i}$ are all measurable w.r.t. the past $\sigma$-algebra $\sigma\left\{f \circ T^{i} \mid i \leq 0\right\}$ hence there is no martingale approximation w.r.t. the natural filtration but there is a martingale approximation w.r.t. another filtration (cf. [V09]).

It remains an open question whether there exists a process $\left(f \circ T^{i}\right)$ regular w.r.t. the natural filtration, without martingale approximation w.r.t. the natural filtration, but with a martingale approximation w.r.t. another filtration $\left(\mathcal{F}_{i}\right)$.

What can be shown for the moment is a process having a martingale approximation both w.r.t. the natural filtration and w.r.t. another filtration $\left(\mathcal{F}_{i}\right)$, and the rate of approximation for $\left(\mathcal{F}_{i}\right)$ is much faster. 
Proposition 3. For any sequences of $a_{n}>0, b_{n}>0,\left(a_{n}\right)$ non decreasing, $b_{n} \searrow 0$, there exists a process $\left(f \circ T^{i}\right)$ and a filtration $\left(\mathcal{F}_{j}\right), m^{\prime} \in L^{2}\left(\mathcal{F}_{0}\right) \ominus L^{2}\left(\mathcal{F}_{-1}\right)$, such that $\left\|S_{n}\left(f-m^{\prime}\right)\right\|_{2} \leq a_{n}$ for all $n \geq 1$, while for the natural filtration $\mathcal{G}_{j}=\sigma\left(f \circ T^{i}\right.$ : $i \leq j)$ and any $m^{\prime \prime} \in L^{2}\left(\mathcal{G}_{0}\right) \ominus L^{2}\left(\mathcal{G}_{-1}\right),\left\|S_{n}\left(f-m^{\prime \prime}\right)\right\|_{2} \geq b_{n} \sqrt{n}$ infinitely many times.

As shown by (e.g.) Wu and Zhao-Woodroofe, a fast rate of martingale approximation implies invariance priniciples and the law of iterated logarithm ([Wu07], $[\mathrm{Z}-\mathrm{Wo}])$. These limit theorems thus can be infered using the filtration $\left(\mathcal{F}_{j}\right)$ but not (in the same way) using the natural filtration $\left(\mathcal{G}_{j}\right)$.

\section{Proofs.}

The proof of Proposition 1 leads to a problem of independent interest which can be expressed as a property of analytic functions, or as a problem in spectral theory; these questions will be treated in the last chapter of the article.

Proof of Proposition 1. As shown in [V93], it is sufficient to prove that for every $m \in L^{2}\left(\mathcal{F}_{0}\right) \ominus L^{2}\left(\mathcal{F}_{-1}\right)$, $\limsup _{n \rightarrow \infty} \frac{1}{\sqrt{n}}\left\|\sum_{i=0}^{n-1}\left(X_{i}-m\right)\right\|_{2}>0$ : if there is no $c>0$ in (1), there is a sequence of $m_{k} \in L^{2}\left(\mathcal{F}_{0}\right) \ominus L^{2}\left(\mathcal{F}_{-1}\right)$ such that $\lim \sup _{n \rightarrow \infty} \frac{1}{\sqrt{n}}\left\|\sum_{i=0}^{n-1}\left(X_{i}-m_{k}\right)\right\|_{2} \rightarrow 0$ as $k \rightarrow \infty$. Then, $m_{k} \rightarrow m \in L^{2}\left(\mathcal{F}_{0}\right) \ominus$ $L^{2}\left(\mathcal{F}_{-1}\right)$ and $\lim _{n \rightarrow \infty} \frac{1}{\sqrt{n}} \| \sum_{i=0}^{n-1}\left(X_{i}-m_{)} \|_{2} \rightarrow 0\right.$.

Let $\left(e_{i}\right)_{i \in \mathbb{Z}}$ be a sequence of $\mathcal{N}(0,1)$ distributed independent random variables, $a_{i}, i \in \mathbb{Z}$, be real numbers, $\sum_{i \in \mathbb{Z}} a_{i}^{2}<\infty, X_{n}=\sum_{i=0}^{\infty} a_{i} e_{n-i} . \quad\left(X_{n}\right)$ is then a stationary linear process. $T$ is a measure preserving and bimeasurable transformation such that $e_{i} \circ T=e_{i+1}$. $U$ is the unitary operator in $L^{2}(\mathcal{A})$ defined by $U f=f \circ T$. We thus have $X_{n}=U^{n} X_{0}=X_{0} \circ T^{n}$. By $\mathcal{F}_{k}$ we denote the filtration $\mathcal{F}_{k}=\sigma\left\{e_{i} \mid i \leq k\right\}$. Notice that $T^{-1} \mathcal{F}_{k-1}=\mathcal{F}_{k}$ and that the process $\left(X_{k}\right)$ is adapted to the filtration $\left(\mathcal{F}_{k}\right)$. We will find the process $\left(X_{k}\right)$ such that there is no martingale approximation w.r.t. $\left(\mathcal{F}_{k}\right)$, and the random variables are mutually orthogonal. Because $\left(X_{k}\right)$ is a Gaussian process, orthogonality implies independence.

Lemma 1 ([V09]). If $m \in L^{2}\left(\mathcal{F}_{0}\right) \ominus L^{2}\left(\mathcal{F}_{-1}\right)$ is such that

$$
\frac{1}{\sqrt{n}}\left\|\sum_{i=0}^{n-1}\left(X_{i}-U^{i} m\right)\right\|_{2} \rightarrow 0
$$

then $m=c e_{0}$ for some $c \in \mathbb{R}$.

Proof of Lemma 1. We have $e_{0} \in L^{2}\left(\mathcal{F}_{0}\right) \ominus L^{2}\left(\mathcal{F}_{-1}\right)$; suppose that $E$ is the Hilbert space generated by $e_{0}$ and $F$ its orthogonal complement in $L^{2}\left(\mathcal{F}_{0}\right) \ominus L^{2}\left(\mathcal{F}_{-1}\right)$, $m=m^{\prime}+m^{\prime \prime}$ where $m^{\prime}=c e_{0} \in E$ for some real number $c$ and $m^{\prime \prime} \in F$. For $f=X_{0}$, the random variables $S_{n}\left(f-m^{\prime}\right)$ and $S_{n}\left(m^{\prime \prime}\right)$ are then mutually orthogonal, hence $\left\|S_{n}\left(m^{\prime \prime}\right)\right\|_{2} \leq\left\|S_{n}(f-m)\right\|_{2}$, therefore $m^{\prime \prime}=0$.

Without loss of generality we can suppose $\left\|X_{0}\right\|_{2}=1$.

Lemma 2 ([V09]). If $X_{i}$ are mutually orthogonal and

$$
\limsup _{n \rightarrow \infty} \frac{1}{\sqrt{n}}\left\|\sum_{i=0}^{n-1}\left(X_{i}-c e_{i}\right)\right\|_{2}=0,
$$


then $|c|=1$.

Proof of Lemma 2. Recall that $\left\|X_{0}\right\|_{2}=1=\left\|e_{0}\right\|_{2}$. We have $\left\|\sum_{i=0}^{n-1}\left(X_{i}-c e_{i}\right)\right\|_{2} \geq$ || $\sum_{i=0}^{n-1} X_{i}\left\|_{2}-\right\| \sum_{i=0}^{n-1} c e_{i} \|_{2}|=| 1-|c| \mid \sqrt{n}$ by independence of $X_{i}$ and of $e_{i}$.

Lemma 3. Let $X_{i}$ be mutually orthogonal. Then

$$
\limsup _{n \rightarrow \infty} \frac{1}{\sqrt{n}}\left\|\sum_{i=0}^{n-1}\left(X_{i}-c e_{i}\right)\right\|_{2}=0
$$

if and only if

$$
\frac{1}{n} \sum_{k=0}^{n-1} \sum_{j=0}^{k} a_{j} \rightarrow c
$$

Proof of Lemma 3. Recall that we assume $\left\|X_{0}\right\|_{2}=1$. By Lemma 2, (1) can take place for $c=1$ or $c=-1$ only. We have

$$
\left\|\sum_{j=0}^{n-1}\left(X_{j}-c e_{j}\right)\right\|^{2}=\left\|\sum_{j=0}^{n-1} X_{j}\right\|^{2}+c^{2}\left\|\sum_{j=0}^{n-1} e_{j}\right\|^{2}-2 c E\left[\left(\sum_{j=0}^{n-1} X_{j}\right)\left(\sum_{j=0}^{n-1} e_{j}\right)\right] ;
$$

because $\left(e_{j}\right)$ is an orthonormal sequence and $X_{k}=\sum_{j=0}^{\infty} a_{j} e_{k-j}$,

$$
\limsup _{n \rightarrow \infty} \frac{1}{\sqrt{n}}\left\|\sum_{j=0}^{n-1}\left(X_{j}-c e_{j}\right)\right\|_{2}=0
$$

if and only if

$$
\left.\frac{1}{n} E\left[\left(\sum_{j=0}^{n-1} X_{j}\right)\left(\sum_{j=0}^{n-1} e_{j}\right)\right]\right]=\frac{1}{n} \sum_{k=0}^{n-1} \sum_{j=0}^{k} a_{j} \rightarrow c
$$

To finish the proof of Proposition 1 it is thus sufficient to find $\left(X_{k}\right)$ such that $X_{k}$ are mutually orthogonal, $\left\|X_{0}\right\|_{2}=1$, and $\frac{1}{n} \sum_{k=0}^{n-1} \sum_{j=0}^{k} a_{j}$ converge neither to 1 , nor to -1 .

Let $H$ be the Hilbert space generated by $e_{i}, i \in \mathbb{Z}$. Let $K=\{z \in \mathbb{C}|| z \mid=1\}$ and $\lambda$ be the Lebesgue probability measure on $K$. By $H^{\prime}$ we denote the Hilbert space $L^{2}(\lambda)$; it is generated by the functions $z^{k}, k \in \mathbb{Z}$. The multiplication $(V f)(z)=z f(z)$ is a unitary operator in $H^{\prime}$. There exists a Hilbert space isomorphism $\phi: H \rightarrow H^{\prime}$ such that $\phi(U v)=V \phi(v)$ for all $v \in H([\mathrm{~A}])$. For any $f \in H^{\prime}$ there thus exists $X=\phi^{-1}(f) \in H ; X=\sum_{i \in \mathbb{Z}} a_{-i} e_{i}$ where $a_{-i}=\hat{f}(i)$ are the Fourier coefficients of $f .\left(X_{k}\right)=\left(U^{k} X\right)$ is thus a gaussian process ([A]). Because $E\left(f U^{k} f\right)=\int_{K} f(z) \overline{z^{k} f(z)} d \lambda(z)=\int_{K} z^{-k} d \lambda(z)$, the condition $|f|=1$ guarantees mutual orthogonality. 
We thus seek a function $f(z)=\sum_{j=0}^{\infty} a_{j} z^{j}$ which is holomorphic on the open unit disc $D=\{z \in \mathbb{C}|| z \mid<1\}, a_{j}$ are all real, $\sum_{j=0}^{\infty} a_{j}^{2}<\infty,|f|=1$ almost everywhere on $K$, and (2) holds neither for $c=1$, nor for $c=-1$. As shown in the next section, such a function exists.

Proof of Proposition 2. Let $\left(e_{i}\right)_{i \in \mathbb{Z}}$ and $\left(f_{i}\right)_{i \in \mathbb{Z}}$ be two independent sequences of iid, each of them taking values \pm 1 with probabilities $1 / 2, g=1+\sum_{i=1}^{\infty} e_{i} / 3^{2 i}+$ $\sum_{i=1}^{\infty} e_{-i} / 3^{2 i+1}, F=f_{0} g+2 e_{0}$.

We denote $\mathcal{E}_{k}=\sigma\left\{e_{i} \mid i \leq k\right\}, \mathcal{F}_{k}=\sigma\left\{f_{i} \mid i \leq k\right\}, \mathcal{C}_{k}=\sigma\left\{e_{i}, f_{i} \mid i \leq k\right\}$, and $\left(\mathcal{G}_{i}\right)$ is the natural filtration of the process $\left(F \circ T^{i}\right)$. Remark that the $\sigma$-algebra generated by $g$ equals $\mathcal{E}_{\infty}$, hence $\mathcal{G}_{i}=\mathcal{F}_{i} \vee \mathcal{E}_{\infty}$, the $\sigma$-algebra generated by $\mathcal{E}_{\infty}$ and $\mathcal{F}_{i}$. For any $i, g$ is $\left(\mathcal{G}_{i}\right)$-measurable and we therefore have $E\left(F \mid \mathcal{G}_{-\infty}\right)=e_{0}$. Any $m \in L^{2}\left(\mathcal{G}_{0}\right) \ominus L^{2}\left(\mathcal{G}_{-1}\right)$ is thus orthogonal w.r.t. the sequence $\left(e_{0} \circ T^{i}\right)$ hence there does not exist any martingale approximation of the process $\left(F \circ T^{i}\right)$ w.r.t. the natural filtration $\left(\mathcal{G}_{i}\right)$. On the other hand, there exists a nice approximation (a martingale-coboundary decomposition) for the filtration $\left(\mathcal{C}_{k}\right)$. To see this, we notice that

- $E\left(f_{0} e_{i} \mid \mathcal{C}_{k}\right)=f_{i} e_{i}$ if $k \geq 0, i \leq k$,

- $E\left(f_{0} e_{i} \mid \mathcal{C}_{k}\right)=f_{0} E\left(e_{i} \mid \mathcal{C}_{k}\right)=0$ if $k \geq 0, i>k$,

- $E\left(f_{0} e_{i} \mid \mathcal{C}_{k}\right)=e_{i} E\left(f_{0} \mid \mathcal{C}_{k}\right)=0$ if $k<0, i \leq k$,

- $E\left(f_{0} e_{i} \mid \mathcal{C}_{k}\right)=E\left(f_{i} e_{i}\right)=0$ if $k<0, i>k$.

We therefore have $E\left(F \mid \mathcal{C}_{k+1}\right)-E\left(F \mid \mathcal{C}_{k}\right)=f_{0} e_{k+1} / 3^{2 k+2}$ for all $k \geq 0, E\left(F \mid \mathcal{C}_{0}\right)-$ $E\left(F \mid \mathcal{C}_{-1}\right)=f_{0} e_{0}+f_{0} \sum_{i=1}^{\infty} e_{-i} / 3^{2 i+1}+2 e_{0}, E\left(F \mid \mathcal{C}_{k}\right)-E\left(F \mid \mathcal{C}_{k-1}\right)=0$ for all $k<0$. This implies that the Hannan's criterium $\sum_{k \in \mathbb{Z}}\left\|E\left(F \mid \mathcal{C}_{k+1}\right)-E\left(F \mid \mathcal{C}_{k}\right)\right\|_{2}<\infty$ (cf. [Ha-He]; [V93] for the non adapted version) is satisfied and there is a martingale approximation.

Proof of Proposition 3. Recall that we denote $U f=f \circ T$. Let $e_{k}, 1 \leq k<\infty$, be random variables with $\left\|e_{k}\right\|_{2}=1$, such that for each $k,\left(U^{i} e_{k}\right)_{i}$ is an iid sequence and $\left(U^{i} e_{k}\right)_{i}$ are mutually independent processes. For each $k, e_{k}$ takes values $\pm q_{k}$ with probabilities $1 / 2 q_{k}^{2}$ (the values of $q_{k}$ will be specified later).

Let $\rho_{k}, p_{k}, k=1,2, \ldots$, be real numbers, $0<\rho_{k} \searrow 0, \rho_{k}<1 / 10,0<p_{k}$, $\sum_{k=1}^{\infty} p_{k}^{2}<\infty .0<\phi(k) \nearrow \infty$ are positive integers. We define

$$
f=\sum_{k=1}^{\infty} p_{k}\left[\rho_{k} e_{k}-\left(1+\rho_{k}\right) U^{-\phi(k)} e_{k}\right]
$$

Notice that the series converges in $L^{2}$ (as we shall see later, the sequence of $q_{n}$ grows exponentially fast hence the series of $f$ converges almost surely). Suppose that the values of $q_{k}, p_{k}, \rho_{k}, \phi(k)$ are such that the random variables $e_{k}$ are $\sigma\{f\}$-measurable (this will be shown at the end of the proof). Therefore, $\mathcal{G}_{j}=\sigma\left\{f \circ T^{i}: i \leq j\right\}=$ $\sigma\left\{e_{k} \circ T^{i}: i \leq j, k=1,2, \ldots\right\} ;\left(\mathcal{G}_{j}\right)$ is the natural filtration of the process $\left(f \circ T^{i}\right)$.

Define $\mathcal{F}_{j}=\sigma\left\{U^{i-\phi(k)} e_{k}: i \leq j, k=1,2, \ldots\right\} .\left(\mathcal{F}_{j}\right)$ is thus a filtration.

Define $m^{\prime}=-\sum_{k=1}^{\infty} p_{k} U^{-\phi(k)} e_{k} \in L^{2}\left(\mathcal{F}_{0}\right) \ominus L^{2}\left(\mathcal{F}_{-1}\right)$. By mutual orthogonality of the processes $\left(e_{k} \circ T^{i}\right)\left(e_{k} \circ T^{i}\right.$ are mutually independent and $\left.E\left(e_{k}\right)=0\right)$ and 
because $\left\|e_{k}\right\|_{2}=1$, we have

$$
\begin{gathered}
\left\|S_{n}\left(f-m^{\prime}\right)\right\|_{2}^{2}=\sum_{k=1}^{\infty} p_{k}^{2} \rho_{k}^{2}\left\|S_{n}\left(e_{k}-U^{-\phi(k)} e_{k}\right)\right\|_{2}^{2}= \\
=2 \sum_{\phi(k) \leq n} p_{k}^{2} \rho_{k}^{2} \phi(k)+2 n \sum_{\phi(k)>n} p_{k}^{2} \rho_{k}^{2} .
\end{gathered}
$$

Define $m^{*}=-\sum_{k=1}^{\infty} p_{k} e_{k} \in L^{2}\left(\mathcal{G}_{0}\right) \ominus L^{2}\left(\mathcal{G}_{-1}\right)$. Then

$$
\begin{gathered}
\left\|S_{n}\left(f-m^{*}\right)\right\|_{2}^{2}=\sum_{k=1}^{\infty} p_{k}^{2}\left(1+\rho_{k}\right)^{2}\left\|S_{n}\left(e_{k}-U^{-\phi(k)} e_{k}\right)\right\|_{2}^{2}= \\
=2 \sum_{\phi(k) \leq n} p_{k}^{2}\left(1+\rho_{k}\right)^{2} \phi(k)+2 n \sum_{\phi(k)>n} p_{k}^{2}\left(1+\rho_{k}\right)^{2} .
\end{gathered}
$$

Because $\phi(k) \nearrow \infty, \sum_{k=1}^{\infty} p_{k}^{2}<\infty$, and $\rho_{k}^{2}<1,\left\|S_{n}\left(f-m^{\prime}\right)\right\|_{2}=o(n)$ and $\left\|S_{n}\left(f-m^{*}\right)\right\|_{2}=o(n)$.

Now, we will find the constants $p_{k}, \rho_{k}, \phi(k)$ so that $\left\|S_{n}\left(f-m^{\prime \prime}\right)\right\|_{2}>b_{n} / \sqrt{n}$ infinitely many times for any $m^{\prime \prime} \in L^{2}\left(\mathcal{G}_{0}\right) \ominus L^{2}\left(\mathcal{G}_{-1}\right)$, and $\left\|S_{n}\left(f-m^{\prime}\right)\right\|_{2} \leq a_{n}$ for all $n$.

For the $p_{k}$ we can choose e.g. $p_{k}=1 / k$. Then we define $\phi(0)=0$ and $\phi(k) \nearrow \infty$ increasing fast enough so that for every $j \geq 1$

$$
2 \sum_{k=j+1}^{\infty} p_{k}^{2}>b_{\phi(j)}^{2}
$$

This implies that $\left\|S_{n}\left(f-m^{*}\right)\right\|_{2} \geq b_{n} \sqrt{n}$ for every $n=\phi(j)$. Notice that for any $m^{\prime \prime} \in L^{2}\left(\mathcal{G}_{0}\right) \ominus L^{2}\left(\mathcal{G}_{-1}\right)$ we have $\left\|S_{n}\left(f-m^{\prime \prime}\right)\right\|_{2} \geq\left\|S_{n}\left(m^{*}-m^{\prime \prime}\right)\right\|_{2}-\left\|S_{n}\left(f-m^{*}\right)\right\|_{2}$ and $\left\|S_{n}\left(m^{*}-m^{\prime \prime}\right)\right\|_{2}=\sqrt{n}\left\|m^{\prime \prime}-m^{*}\right\|_{2}$ hence, if $\left\|m^{\prime \prime}-m^{*}\right\|_{2}>0,\left\|S_{n}\left(f-m^{\prime \prime}\right)\right\|_{2}>$ $c \sqrt{n}$ infinitely many times for some $c>0$.

Next we find $\rho_{k}$ small enough so that $\left\|S_{n}\left(f-m^{\prime}\right)\right\|_{2} \leq a_{n}$ for all $n \geq 1$. Without loss of generality we can suppose that $a_{n}=1$ for all $n$. We take $\rho_{k}^{2}=1 / 8 \phi(k)$, for all $k$. Recall that $\sum_{k=1}^{\infty} 1 / k^{2}<2$. Then

$$
2 \sum_{k=1}^{\infty} p_{k}^{2} \rho_{k}^{2} \phi(k)=\frac{1}{4} \sum_{k=1}^{\infty} \frac{1}{k^{2}}<1 / 2
$$

and

$$
2 n \sum_{\phi(k)>n} p_{k}^{2} \rho_{k}^{2}<\frac{1}{4} \sum_{\phi(k)>n} \frac{1}{k^{2}}<1 / 2, \quad n=1,2, \ldots,
$$

hence $\left\|S_{n}\left(f-m^{\prime}\right)\right\|_{2} \leq 1$.

Eventually we choose the numbers $q_{k}$ so that the functions $e_{k}$ are $\sigma\{f\}$-measurable. Denote

$$
\begin{gathered}
f_{n}=\sum_{k=1}^{n} p_{k}\left[\rho_{k} e_{k}-\left(1+\rho_{k}\right) U^{-\phi(k)} e_{k}\right], \\
r=r_{n}=3 \sum_{k=1}^{n} p_{k} q_{k}, \quad s=s_{n+1}=10 r / \rho_{n+1}>100 r .
\end{gathered}
$$


Notice that the previous calculation does not impose any condition on the values of $q_{k}$ and that $\left|f_{n}\right| \leq r_{n}$. We define the numbers $q_{k}$ so that $q_{1}=1$ and

$$
10 r_{n}=30 \sum_{k=1}^{n} p_{k} q_{k}=\rho_{n+1} p_{n+1} q_{n+1}, \quad n=1,2, \ldots
$$

We will show that for $x, y \in\left\{-q_{n+1}, 0, q_{n+1}\right\}$ the sets $\left\{e_{n+1}=x, U^{-\phi(n+1)} e_{n+1}=\right.$ $y\}$ are $\sigma\left\{f_{n+1}\right\}$-measurable. By definition we get the inclusions

$$
\begin{aligned}
& \left\{e_{n+1}=-q_{n+1}, U^{-\phi(n+1)} e_{n+1}=-q_{n+1}\right\} \subset f_{n+1}^{-1}((s-r, s+r)), \\
& \left\{e_{n+1}=-q_{n+1}, U^{-\phi(n+1)} e_{n+1}=0\right\} \subset f_{n+1}^{-1}((-11 r,-9 r)), \\
& \left\{e_{n+1}=-q_{n+1}, U^{-\phi(n+1)} e_{n+1}=q_{n+1}\right\} \subset f_{n+1}^{-1}((-s-21 r,-s-19 r)), \\
& \left\{e_{n+1}=0, U^{-\phi(n+1)} e_{n+1}=-q_{n+1}\right\} \subset f_{n+1}^{-1}((s+9 r, s+11 r)), \\
& \left\{e_{n+1}=0, U^{-\phi(n+1)} e_{n+1}=0\right\} \subset f_{n+1}^{-1}((-r, r)), \\
& \left\{e_{n+1}=0, U^{-\phi(n+1)} e_{n+1}=q_{n+1}\right\} \subset f_{n+1}^{-1}((-s-11 r,-s-9 r)), \\
& \left\{e_{n+1}=q_{n+1}, U^{-\phi(n+1)} e_{n+1}=-q_{n+1}\right\} \subset f_{n+1}^{-1}((s+19 r, s+21 r)), \\
& \left\{e_{n+1}=q_{n+1}, U^{-\phi(n+1)} e_{n+1}=0\right\} \subset f_{n+1}^{-1}((9 r, 11 r)), \\
& \left\{e_{n+1}=q_{n+1}, U^{-\phi(n+1)} e_{n+1}=q_{n+1}\right\} \subset f_{n+1}^{-1}((-s-r,-s+r)) .
\end{aligned}
$$

Because the sets on the left give a partition of $\Omega$ and the sets on the right are mutually disjoint, the inclusions are equalities, hence the sets $\left\{e_{n+1}=x, U^{-\phi(n+1)} e_{n+1}=\right.$ $y\}$ are $\sigma\left\{f_{n+1}\right\}$-measurable. Therefore, the functions $e_{n+1}$ and $U^{-\phi(n+1)} e_{n+1}$ are $\sigma\left\{f_{n+1}\right\}$-measurable, $n \geq 1$.

Recall $f_{1}=p_{1}\left[\rho_{1} e_{1}-\left(1+\rho_{1}\right) U^{-\phi(1)} e_{1}\right]$ where $p_{1}=1=q_{1}, \rho_{1}<1 / 10, e_{1}= \pm 1$ with probabilities $1 / 2$. We deduce that the functions $e_{1}$ and $U^{-\phi(1)} e_{1}$ are $\sigma\left\{f_{1}\right\}-$ measurable.

Because the functions $e_{n+1}$ and $U^{-\phi(n+1)} e_{n+1}$ are $\sigma\left\{f_{n+1}\right\}$-measurable, $f_{n}=$ $f_{n+1}-p_{n+1}\left[\rho_{n+1} e_{n+1}-\left(1+\rho_{n+1}\right) U^{-\phi(n+1)} e_{n+1}\right]$ is $\sigma\left\{f_{n+1}\right\}$-measurable. Using $\sigma\left\{f_{n}\right\}$-measurability of $e_{n}$ and $U^{-\phi(n)} e_{n}$ we deduce that $e_{n}$ and $U^{-\phi(n)} e_{n}$ are $\sigma\left\{f_{n+1}\right\}$-measurable. By induction we deduce that all $e_{k}$ (and $U^{-\phi(k)} e_{k}$ too) with $1 \leq k \leq n+1$ are $\sigma\left\{f_{n+1}\right\}$-measurable.

The $q_{n}$ are growing exponentially fast hence the measures of the supports of $e_{n}$ are decreasing exponentially fast. For $F_{n}=\cup_{k=n}^{\infty}\left\{e_{k} \neq 0\right\}$ we thus have $\mu\left(F_{n}\right) \searrow$ 0 . For $E_{n}=\Omega \backslash F_{n}$ we have $E_{n} \subset E_{n+1}$ for all $n$ and $\mu\left(E_{n}\right) \nearrow 1$. Because $f_{n}=f$ on $E_{n}, f_{n}^{-1}(B) \cap E_{n}=f^{-1}(B) \cap E_{n}$ for every Borel set $B \subset \mathbb{R}$. Therefore, $\sigma\left\{e_{k}\right\} \cap E_{n} \subset \sigma\{f\} \cap E_{n}, 1 \leq k \leq n, n=1,2, \ldots$. We deduce that all the functions $e_{k}, k \geq 1$, are $\sigma\{f\}$-measurable.

\section{A bad approximation property.}

In the preceding section, the problem of finding a gaussian, adaptated process $\left(X_{k}\right)$, which is in some sense (described before) badly approximable, was reduced to the following, function-theoretical, question, in which one sets

$$
f(z)=\sum_{n=0}^{\infty} a_{n} z^{n}, A_{n}=\sum_{j=0}^{n} a_{j}, M_{n}=\frac{A_{0}+\ldots+A_{n-1}}{n}, f^{*}\left(e^{i t}\right)=\lim _{\substack{<\\ r \rightarrow 1}} f\left(r e^{i t}\right),
$$

the function $f$ being a bounded, analytic function in the open unit disk $D$ of the complex plane, with real coefficients $a_{n}$, and $f^{*}\left(e^{i t}\right)$ denoting its radial limits, which 
exists almost everywhere with respect to the Lebesgue measure, according to a wellknown result (Fatou's theorem: see [DU] p.6 or [RU] p.340). If this radial limit has modulus one (a.e.), the function $f$ is said to be inner (see [DU] p.24 or [RU] p.342), which is a severe restriction on its behavior. But this is this "innerness" property which has guaranteed us the orthogonality, therefore the mutual independence, of the $X_{k}^{\prime} s$. And the problem now is :

Problem Find an inner function $f$ with real coefficients such that one of the following cases occurs :

(1) $M_{n}$ has no limit,

(2) $M_{n}$ has a limit $c$, but $c \neq \pm 1$.

This turns out to be possible in many ways. We just indicate two examples below, of a different nature.

Example 1. Let $a>0$ and $f(z)=e^{-a\left(\frac{1+z}{1-z}\right)}$. This is clearly a zero-free inner function (called singular) with real coefficients $a_{n}$, and with $f^{*}\left(e^{i t}\right)=e^{-i a \operatorname{cotg} \frac{t}{2}}$. Now, it was proved in $[\mathrm{NESH}]$ that we have

$$
a_{n}=\pi^{-\frac{1}{2}}(2 a)^{\frac{1}{4}} n^{-\frac{3}{4}} \cos \left(2 \sqrt{2 a n}+\frac{\pi}{4}\right)+O\left(n^{-\frac{5}{4}}\right)
$$

from which it easily follows, using summation by parts, that $A_{n}$ converges, necessarily to 0 by Abel's theorem, since the radial limit of $f$ at 1 is clearly 0 . (Another approach to that example is given in [BAKOQU] where it is observed that $A_{n}=e^{-a} L_{n}(2 a)$, where $L_{n}$ is the $n$-th Laguerre polynomial, so that $\left.A_{n} \rightarrow 0\right)$. Therefore, $M_{n} \rightarrow 0$, i.e. $M_{n}$ has a limit $c$, but this limit does not have the correct value $c= \pm 1$, so that the corresponding gaussian process is badly approximable.

Example 2. Let $\left(z_{n}\right)_{n \geq 1}$ be a sequence of real numbers between 0 and 1 , such that $\sum_{n=1}^{\infty}\left(1-z_{n}\right)<\infty$ (a Blaschke sequence) and let $B$ be the corresponding Blaschke product, namely

$$
B(z)=\prod_{n=1}^{\infty} \frac{z_{n}-z}{1-z_{n} z} .
$$

It is well-known $\left([\mathrm{RU}]\right.$ p.312) that this is an inner function. Assume that $M_{n}$ has a limit $c$. Then, by a well-known extension (due to Frobenius) of Abel's theorem ([KOR] p.4), we have $\lim _{r\lrcorner_{1}} B(r)=c$. This last fact may quite well happen, taking for example $z_{n}=1-n^{-\alpha}, \alpha>1$ ([RU] p.317). But then, necessarily, $c=0$ since $B\left(z_{n}\right)=0$ and $z_{n} \rightarrow 1$. Observe that a finite Blaschke product, even with complex zeros symmetric with respect to the real axis, would never do the job, since then $B$ extends analytically across the closed unit disk, and $A_{n} \rightarrow B(1)=1$, so that $M_{n} \rightarrow 1$. (This might be the only case of an inner function with real coefficients for which $\left.M_{n} \rightarrow 1\right)$. Finally, if $M_{n}$ has no limit, we automatically have an example of a badly approximable gaussian process. We show that this can happen on a simple specific example:

Proposition 6. There exists an infinite Blaschke product $B(z)=\sum_{n=0}^{\infty} a_{n} z^{n}$ with real zeros and coefficients, verifying the two following conditions :

(1) $\liminf \underset{r \rightarrow 1}{<}|B(r)|=0$ and $\lim \sup _{r \rightarrow 1}^{<}|B(r)|>0$;

(2) $A_{n}=a_{0}+\ldots+a_{n}$ is not Cesàro-summable, i.e. $M_{n}$ has no limit. 
Proof. Observe that 2. is an automatic consequence of 1., through the Frobenius theorem already mentioned. We now take

$$
z_{n}=1-2^{-n}
$$

Let $r=\frac{z_{n}+z_{n+1}}{2}$, and proceed to minorize $B(r)$. We have $|B(r)|=P_{1} P_{2} P_{3} P_{4}$, where

$$
P_{1}=\prod_{j<n} \frac{r-z_{j}}{1-z_{j} r} ; P_{2}=\frac{r-z_{n}}{1-z_{n} r} ; P_{3}=\frac{-r+z_{n+1}}{1-z_{n+1} r} ; P_{4}=\prod_{j>n+1} \frac{-r+z_{j}}{1-z_{j} r}
$$

Now, we see that

(1)

$$
\begin{gathered}
P_{1} \geq \prod_{j<n} \frac{z_{n}-z_{j}}{1-z_{j} z_{n}} \geq \prod_{j<n} \frac{2^{-j}-2^{-n}}{2^{-j}+2^{-n}}=\prod_{k<n} \frac{1-2^{-k}}{1+2^{-k}} \\
\geq \prod_{k=1}^{\infty} \frac{1-2^{-k}}{1+2^{-k}}=c>0 .
\end{gathered}
$$

(2) One has similarly $P_{4} \geq c>0$.

(3)

$$
P_{2}=\frac{z_{n+1}-z_{n}}{2-z_{n}\left(z_{n}+z_{n+1}\right)} \geq \frac{z_{n+1}-z_{n}}{2\left(1-z_{n}^{2}\right)} \geq \frac{1}{4} \frac{z_{n+1}-z_{n}}{\left(1-z_{n}\right)}=\frac{1}{8}
$$

(4) One has similarly $P_{3} \geq \frac{1}{8}$.

This ends the proof of Proposition 6.

\section{$\underline{\text { Remarks : }}$}

(1) The specific sequence $z_{n}=1-2^{-n}$ in the proof of the proposition verifies the Newman lacunarity condition:

$$
\frac{1-\left|z_{n+1}\right|}{1-\left|z_{n}\right|} \leq \rho<1
$$

Such sequences will in particular be interpolation sequences in the sense of Carleson ([DU] p.149 or [HOF] p.203), and the Taylor coefficients of $a_{n}$ of the corresponding Newman-lacunary Blaschke product will verify ([NESH]):

$$
a_{n}=O\left(\frac{1}{n}\right)
$$

(2) It can be proved that, for any infinite Blaschke product, one has

$$
\varlimsup_{n \rightarrow \infty} n\left|a_{n}\right|>0
$$

(see $[\mathrm{NESH}])$. Therefore, the estimate $a_{n}=O\left(\frac{1}{n}\right)$ is the best possible.

(3) The estimate on the Taylor coefficients of a Blaschke product with Newmanlacunary zeros was recently used by Bourgain and Kahane ([BOKA]). 


\section{REFERENCES}

[A] Ash, R., Topics in Stochastic Processes, Acad. Press, New York, 1975.

[BAKOQU] Bayart F., Konyagin S.V., Queffélec H., Real Analysis Exchange 33 (2004), 1-31.

[BOKA] Bourgain J., Kahane J.P., to appear, Ann.Inst.Fourier (2009).

[DeM] Dedecker, J. and Merlevède, F., Necessary and sufficient conditions for the conditional central limit theorem, Ann. Probab. 30 (2002), 1044-1081.

[DU] Duren, P.L., Theory of $H^{p}$-spaces, Dover Publications, Inc., 2000.

[Go] Gordin, M.I., The central limit theorem for stationary processes, Soviet Math. Dokl. 10 (1969), 1174-1176.

[Ha-He] Hall, P. and Heyde, C.C., Martingale Limit Theory and its Application, Academic Press, New York, 1980.

[HOF] Hoffman, K., Banach spaces of analytic Functions, Prentice-Hall, Englewood Cliffs, New Jersey, 1962.

[KOR] Korevaar, J., Tauberian Theory, a Century of Developments, Springer, 2004.

[M-Wo] Maxwell, M. and Woodroofe, M., Central limit thoerems for additive functionals of Markov chains, Ann. Probab. 28 (2000), 713-724.

[NESH] Newman D., Shapiro H., The Taylor coefficients of inner functions, Mich.Math.J. 2 (1962), 249-255.

[Pe-U] Peligrad, M. and Utev, S., A new maximal inequality and invariance principle for stationary sequences, Ann. Probab. 33 (2005), 798-815.

[RU] Rudin W., Real and Complex Analysis, third edition, McGraw-Hill, 1987.

[V85] Volný, D., Determinism and martingale decomposition of strictly stationary sequences, Proceedings of the 13th Winter School on Abstract analysis, Suppl. si Rend. di Circ. Mat. di Palermo, Serie II - numero 10 (1985), 185-192.

[V92] Volný, D., The central limit problem for composed arrays of martingale differences, Statistics \& Decisions 10 (1992), 281-290.

[V93] Volný, D., Approximating Martingales and the CLT for Strictly Stationary Processes, Stoch Proc. Appl. 44 (1993), 41-74.

[V09] Volný, D., Martingale approximations of stochastic processes depending on the choice of the filtration, an unpublished result (2009).

[Wu-Wo] Wu, W. B. and Woodroofe, M., Martingale approximation for sums of stationary processes, Ann. Probab. 32 (2004), 1674-1690.

[Wu05] Wu, W. B. Nonlinear system theory: Another look at dependence, Proceedings of the National Academy of Sciences USA 102 (2005), 14150-14154.

[Wu07] Wu W. B., Strong invariance principles for dependent random variables, Annals of Probability 35 (2007), 2294-2320.

[Z-Wo] On martingale approximations, Annals of Applied Probability 18 (2008), 1831-1847. 